ARS María Iñigo Clavo*

ano 15

ก. 30

\title{
¡Sucia Copia!
}

palavras-chave:

Tropicália, Hélio Oiticica, Homi Bhabha, mimesis, ambivalencia, hibridación

keywords:

Tropicália, Hélio Oiticica, Homi Bhabha, mimesis, ambivalence and hibridation

* Professora da Universidade Oberta de Catalonya [UOC].

DOI: 10.11606/issn.2178-0447. ars.2017.134105
Este texto realiza una relectura de la obra Tropicália, de Hélio Oiticica, desde la perspectiva post-colonial para revisar la versión nacional(ista) de la interpretación del trabajo y de la figura de Oiticica; para ello, se centra en una comparativa de los conceptos clave para Homi Bhabha, mimesis, ambivalencia e hibridación, en comparación con la propuesta tropicalista del artista en su atención a la favela de Rio de Janeiro, local "casi igual pero no exatamente" a la ciudad moderna occidental.

This text presents a re-reading of the artwork Tropicália from the point of view of a post-colonial theorization to review the previous national(ist) version of Oiticica's work interpretation and of his figure. For that it focuses in the comparison of several concepts crucial for Homi Bhabha: mimesis, ambivalence and hybridization in comparison with the tropicalist proposal of the artist in his interest of Rio de Janeiro favelas, this place "quite the same, but not really" of the occidental modern city. 
Quizás fue Hélio Oiticica quien mejor supo sintetizar y positivizar muchos de los debates que tuvieron lugar durante los años 60 en Brasil. Esto se refleja en toda su obra de esta década y se cristaliza específicamente en el Esquema Geral da Nova Objetividade y Tropicália, trabajo que expuso por primera vez en 1967. Tropicália es la puerta que separa el arte de los años 60 de un nuevo lenguaje artístico que tomó fuerza desde principios de los años 70 y que se ha equiparado al arte conceptual. Reúne preocupaciones que giran en torno al movimiento neoconcreto, el Pop, el conceptual, lo nacional, lo internacional, la relación vanguardia-política, lo popular y el compromiso intelectual con el pueblo, el desarrollo, la alteridad, la tropicalidad, la antropofagia, etc., términos hasta entonces debatidos y algunos nuevos anticipados. La obra se presenta un año antes del AI-5 (Ato Institucional 5), justo antes del momento en que la explosión intelectual nacional de izquierda de los años 60 comenzara un profundo proceso de transformación. La instalación se componía de dos Penetrables (en portugués, Penetráveis): PN2 "A pureza é um mito" y PN3 "Imagético".

Pero lo primero es la ambientación del espacio. Para entrar en el recinto de la instalación el espectador debía quitarse los zapatos para andar sobre arena y gravas. Con ello participaba de la experiencia de "tocar la tierra", idea fundamental desde sus primeras experiencias en la favela de la Mangueira donde Oiticica descubrió que esa forma de vida había conectado a la ciudad nuevamente, dice Oiticica: "me parecía al caminar por el recinto, por el escenario de Tropicália, estar doblando las quebradas del morro, tan orgánicas como la arquitectura fantástica de las favelas; otra vivencia: la de 'estar pisando la tierra otra vez"'. Con los pies descalzos el espectador se relacionaba con la obra más allá de la pura mirada, sintiendo la arena, las piedras, al andar sobre los Penetrables. Estos estaban rodeados de plantas, y en sus primeros días contaban con un Wakamayo que andaba suelto por la instalación.

El primer Penetrable se componía de una pequeña cabina en que aparecía escrita la frase "a pureza é um mito". Adherida a la puerta había una bolsa que contenía material orgánico/pigmento. La cabina era de color rojo fuerte, al tiempo que el pigmento denotaba la presencia material y táctil del color de sus Bólidos (en portugués, Bólides). El segundo Penetrable, PN3 "Imagético", es un espacio construido con telas coloridas, estampadas y maderas, formando un camino en espiral cuadrada que llega hasta el centro del espacio, cada vez más y más oscuro. Conforme el espectador avanza hacia el estrecho pasillo el Penetrable se va ensombreciendo hasta desembocar en un luminoso monitor de televisión que parece retransmitir una imagen en directo.
88

María Iñigo Clavo

¡Sucia Copia!

1. OITICICA, Hélio. Aspiro ao grande labirinto. Rio de Janeiro: Rocco, 1986, p. 107, traducción mía. "El ambiente creado era obviamente tropical, como el de un mundo de casa de campo y, lo más importante, había una sensación de estar de nuevo 'pisando la tierra'. Esta sensación la sentía yo anteriormente al caminar por los morros, por la favela, e incluso el recorrido de entrar, salir, doblar 'por las quebradas' de Tropicália me recuerda mucho a las caminatas por el morro". 
Decir "a pureza é um mito" es una evocación más que conscien-

2. Ibidem, p. 106-108. Dice Oiticica en 1967, "por eso creo que Tropicália, que encierra todo esta serie de proposiciones, vino a contribuir fuertemente a esa objetivación de una imagen brasileña total, para el derrumbe del mito universalista de la cultura brasileña, toda copiada de Europa y de América del Norte, un ariarismo inadmisible aquí: en verdad, quise crear con Tropicália el mito de mestizaje -somos negros, indios, blancos, todo al mismo tiempo- nuestra cultura nada tiene que ver con

la europea, a pesar de estar hasta hoy a ella sometida: solo el negro y el indio no cedieron a ella. Quien no tenga conciencia de esto se queda fuera. Para la creación de una verdadera cultura brasileña, característica y fuerte, expresiva al menos, esa maldita herencia europea y americana tendrá que ser absorbida, antropofágicamente, por la negra e india de nuestra tierra, que en verdad son las únicas significativas, pues la mayoría de los productos del arte brasileño son híbridos, intelectualizada al extremo, vacíos de significado propio".

3. Oiticica defiende en los años 1960/70 una visión esencialista de Brasil, pero en 1973 en su texto "Brasil Diarrea" matiza: "en vez de una postura conformista, que se base siempre sobre valores generales absolutos, esa postura constructiva surge de una ambivalencia crítica. Según lo explicaba líneas te de Oiticica a la antropofagia. En los años 60 esta pierde el carácter etnográfico de los años 20 para reforzar la metáfora nacionalista de la autodefinición, pero esta pérdida es una de las causas que permitirá diez años después que la presencia política del Otro comience a hacerse realidad. Este asunto de lo inauténticamente auténtico brasileño, la búsqueda de una "imagen brasileña total" y la alusión a la antropofagia, es el síntoma de la búsqueda en torno a lo nacional que ocupa toda la década de los años 60 en Brasil tanto para la derecha como para la izquierda. Oiticica estuvo muy influenciado por la misma "búsqueda del pueblo", por crear una caracterización de este. "Tropicália es - dice Oiticica - la primerísima tentativa consciente, objetiva, de imponer una imagen obviamente brasileña al contexto actual de vanguardia y de manifestaciones en general del arte nacional" ${ }^{2}$.

Homi Bhabha distingue dos tiempos en el nacionalismo; el pedagógico y el performático, el que se repite en el tiempo y el que se reproduce en el presente. Este último ha de legitimar el discurso nacional construido, ha de corroborarlo, pero al mismo tiempo puede ponerlo en un aprieto por evidenciar sus contradicciones. El proyecto de Oiticica se sitúa entre estos dos espacios: por una parte la contribución de un imaginario brasileño, por otra, su gesto se vuelve irreverente a la hora de aludir a la favela, al povão y a la pobreza, volver al "contacto con la tierra", todo ello podría ser una forma de cuestionar la representación del progreso, de la capitalización brasileña y de la postal turística.

Parece que Oiticica quiere derrumbar una imagen de la brasilidad para enarbolar otra, una "imagen brasileña total" que en efecto no puede menos que alertarnos sobre un proyecto macro-de-representación ${ }^{3}$. Sin embargo de alguna forma, la precariedad de Tropicália está proponiendo una brasilidad que desmonta los estereotipos típicos del exotismo, de la representación tropical de Brasil que tanto el nacionalismo, y por extensión el turismo, trataban de desarrollar. Pero su estratégica es más compleja. El uso de materiales pobres, la reivindicación del espacio de la favela, la arena, las telas, las plantas en macetas, todo ese escenario manual se nos desvela como una réplica deslucida del exotismo turístico y flamante del progreso, casi como una desacreditación. Se separa de su referente como copia deslucida, una "copia sucia". Y esa estrategia es considerada por Bhabha el modo de resistencia por excelencia de los dominados en el espacio colonial. Un modo inconsciente pero con un gran poder de reconfigurar los discursos occidentales que han experimentado la colonización, que los 
evidencia definitivamente como híbridos de su referente cívico de la ciudad moderna, como otra cosa.

Uno de los trabajos cruciales de Bhabha, El lugar de la cultura (2002), tiene que ver con mostrar cómo las proyecciones, usos, instrumentalizaciones, deseos, estereotipos, etc., que el colonizador/dominador arroja sobre el colonizado/dominado no son sino un retrato constante del mismo dominador, y al mismo tiempo una operación de nombrar la diferencia que pone en evidencia el discurso colonial cada vez. Por intermedio de los estudios de Freud y Fanon observa como estas miradas "virtualmente devueltas" posibilitan reconocer en sí mismas el motor de sus acciones, sus miedos y terrores en cada definición y toma de posición ante el Otro. El proceso que Bhabha muestra desde el psicoanálisis tiene lugar en el ámbito del colonizador/dominador, que se verá desencajado en cada gesto por su intento fallido de posicionarse con sus premisas iniciales modernas civilizatorias, y siempre de forma ambivalente, ante la colonización.

El primer concepto repensado por Bhabha es el estereotipo, cuya función es la de crear fijezas sobre el Otro que marcarán las diferencias culturales, históricas y raciales en el discurso del colonialismo. El estereotipo es una "forma de conocimiento e identificación que vacila entre lo que 'siempre está en su lugar', ya conocido, y algo que debe ser repetido ansiosamente" ${ }^{4}$. Es decir que lejos de ser la categoría de lo inamovible y una representación concluida del Otro, se consolida como una necesidad de corroborar compulsivamente determinados conceptos sobre el otro, sobre todo va a delatar una inseguridad constante en esta relación colono/colonizado, una confirmación, una ratificación necesaria para certificar la diferencia colonial cada vez. Lacan entiende lo traumático como un encuentro fallido con lo real, y en cuanto fallido escapa de la representación. En todo caso, lo real aquí tan sólo podrá ser repetido, reproducido una y otra vez ${ }^{5}$, pero no representado. El estereotipo, como repetición ansiosa, por tanto, también podría delatar el encuentro fallido con la realidad del Otro. En otras palabras, más que mostrar certeza, delata una inseguridad que constantemente ha de ser superada. Esta neurosis además se consolida por la naturaleza del estereotipo, que no es unívoco, sino ambivalente.

La ambivalencia es otro de los conceptos centrales del pensamiento de Bhabha. En la medida en que se reconozca la ambivalencia del estereotipo se comprenderá también uno de los cimientos del discurso del poder colonial: una relación de odio y deseo al mismo tiempo. Este desvela la relación entre el discurso y la política,

\section{María Iñigo Clavo}

¡Sucia Copia!

atrás: Es necesario entender que una posición crítica implica unas ambivalencias inevitables: ser apto para juzgar, juzgarse, optar, crear, es estar abierto a ambivalencias, ya que los valores absolutos tienden a castrar cualquiera de estas libertades; incluso diría: pensar siempre en términos absolutos es caer constantemente en error; envejecer fatalmente; adquirir una postura conservadora (conformismos; paternalismos etc.); lo cual no significa que no se deba optar con firmeza: la dificultad de una opción fuerte siempre es la de asumir ambivalencias y separar pieza por pieza cada problema. Asumir ambivalencias no significa aceptar conformísticamente todo ese estado de las cosas". OITICICA, Hélio. Brasil diarréia. In: FUNDACIÓ ANTONI TAPIES. Hélio Oiticica. Barcelona: Fundació Antoni Tapies, 1992.

4. BHABHA, Homi. El lugar de la cultura. Buenos Aires: Manantial, 2002, p. 91.

5. FOSTER, Hal. El retorno de lo real. Madrid: Akal, 2001, p. 136. 
Para comprender la productividad del poder colonial es crucial construir su régimen de verdad, no someter sus representaciones a un juicio normalizador. Sólo entonces se vuelve posible comprender la ambivalencia productiva del objeto del discurso colonial; esa "otredad" que es a la vez un objeto de deseo y de irrisión, una articulación de la diferencia contenida dentro de la fantasía de origen y de identidad. Lo que revela esa lectura son los límites del discurso colonial y permite una trasgresión de esos límites desde el espacio de esa otredad. ${ }^{6}$

Esta idea de ambivalencia ayudará a explicar una de las operaciones más transgresoras del colonizado hacia la autoridad colonial, que trata de legitimarse cada vez intentando mantener sus ideales primigenios. La operación de resistencia ni siquiera es resultado de una acción coordinada de los sometidos, sino un reclamo de la autoridad colonial que acabará por volverse en su contra: la mimesis.

La mimesis tiene lugar cuando el colonizador, queriendo reformar, civilizar y normalizar al Otro, le interpela la adopción de sus formas, ideales, valores, etc. (aprender inglés, convertirle al cristianismo, a introducirse en la civilización, entre otras). Este Otro se desvelará como una copia marchita, lo que Bhabha ha expresado como "casi lo mismo pero no exactamente". El Otro transformado de forma defectuosa en el Mismo acabará por desvelar una vez más la ambivalencia de lo que siendo igual no consigue ser exactamente lo mismo. De esta forma la autoridad colonial se apropia del Otro regulando, disciplinando su alteridad, pero al mismo tiempo el colonizado se convierte en "el signo de lo inapropiado, una diferencia u obstinación que cohesiona la función estratégica dominante del poder colonial, intensifica la vigilancia, y proyecta una amenaza inmanente del poder colonial, tanto sobre el saber "normalizado" como sobre los poderes disciplinados"7.

Por eso el mimetismo, si bien es alienante para el colonizado también lo es para el colonizador puesto que "produce" un nuevo saber, una nueva identidad, un nuevo lugar de la cultura a partir de sus propias normas de civilización reapropiadas, efectivamente como en la operación antropofágica. Este nuevo sujeto se convierte en doble móvil, desestabiliza la identidad inicial y los valores mismos que trata de enarbolar. La mimesis no es sino el paso previo para la hibridación, primero identitaria, para luego ocupar "el lugar de la cultura".

Esta representación/reconocimiento del Otro por ser parcial va a ser denominado por Bhabha "metonimia de la presencia". La visibilidad se produce en el lugar de la interdicción, "entre lo conocido y permisible y lo que, aunque conocido debe ser mantenido oculto; un discurso 
proferido entre líneas y como tal a la vez contra las reglas y dentro de

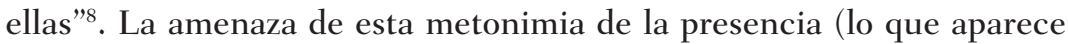
en la operación de estereotipos: "Negro simio, Asiático mentiroso") es su capacidad de crear "'efectos de identidad' conflictivos, fantásticos, discriminatorios, en el ejercicio de un poder que es elusivo porque no oculta esencia alguna, ningún 'sí mismo”’’. Es en este ámbito en que se da la discriminación. Pero

la ambivalencia del mimetismo (casi pero no exactamente) sugiere que la cultura colonial fetichizada es potencial y estratégicamente una constraapelación insurgente: Lo que he llamado sus "efectos de identidad" están siempre crucialmente escindidos. Bajo cubierta de camuflaje, el mimetismo, como fetiche, es un objeto parcial que revalúa radicalmente los conocimientos normativos de la prioridad de raza, escritura, historia. Pues el fetiche imita las formas de autoridad hasta el punto en que las desautoriza. De modo similar, el mimetismo rearticula la presencia en términos de su "otredad", aquello que reniega. ${ }^{10}$

En términos de Oiticica la cultura brasileña sería "un otro espacio", precisamente híbrido: el de la favela. Al igual que con los Parangolés no se puede separar este trabajo, de su experiencia en la favela de la Mangueira, de su fascinación por la deriva que el caos del carnaval produce, por su laberíntica constitución que invita a perderse, que es antropófaga porque te engulle en su interior, para, como explica Paola Berenstein Jacques, encontrarse a uno mismo: pues el objeto último del laberinto será la desorientación, el proceso de pérdida de referencia ${ }^{11}$, el fin de los opuestos.

Su propuesta no es la arquitectura precaria, sino otros modos de habitar y de pensar la vida. La favela crece tal como el matorral brasileño en los espacios desocupados de la ciudad hasta sobrepasarlos y desbordarlos. Precisamente por eso entre 1964 y 1974 el gobierno llevó a cabo un plan de traslado de favelas que estaban ocupando las partes más nobles de la ciudad. Se calcula que fueron destruidas 26.123 casas y 139.218 personas fueron trasladadas a la fuerza.

La defensa que Jacques realiza en su libro Estética da ginga ${ }^{12}$ tiene que ver con este lugar intersticial que la favela ocupa no solo en la ciudad, sino también en los valores de la urbanidad, tema que también Bhabha planteó en el capítulo "Astuta urbanidad". En él describe como las aspiraciones de los colonos con respecto a la creación de la ciudad y su sociedad están constantemente asaltadas por el híbrido y que evidencia la realidad colonial.

\section{María Iñigo Clavo}

¡Sucia Copia!
8. Ibidem, p. 116.
9. BHABHA, Homi. Loc. cit.

10. Ibidem, p. 117.

11. “La idea del laberinto es, por tanto más del orden de la experiencia subjetiva que la del propio laberinto como objeto, a pesar de que ambos están inevitablemente unidos. Así como la noción de fragmento -que considera más importante lo fragmentario que el propio fragmento- en la noción de laberinto, interesa más lo laberíntico que el laberinto como forma. Es más relevante la idea de laberinto (desorientación) del ser laberintado (que recorre el laberintol, que la del laberinto formal (espacio físico). La idea de laberinto es la del espacio laberíntico, relacionado no necesariamente a la forma del laberinto, sino sobre todo, a la experiencia de penetrar en él". JACQUES, Paola. Estética da ginga: arquitetura das favelas através da obra de Hélio Oiticica. Rio de Janeiro: Casa da Palavra, 2001, p. 93.

12. Ibidem, p. 150. 
La respuesta del nativo muestran el continuo deslizamiento entre la inscripción civil y la interpelación colonial. La incertidumbre generada por esa resistencia cambia la demanda misma de la narración. Lo que era dicho dentro de los órdenes de la urbanidad ahora accede al significante colonial. ${ }^{13}$

Es decir que el Otro con su presencia y su forma de habitar siempre está interrogando a la urbanidad dominante. Las piedras preciosas de la civilización y la ciudad, la sociedad civil o el Estado de derecho en la favela no será más que un intento fallido, un constante reproche a las oligarquías. Dice Bhabha:

En sentido piscoanalítico, "imitar" es aferrarse a la negación de las limitaciones del yo (ego); "identificarse" es asimilar conflictivamente. Entre ellos, donde la letra de la ley no será asignada como signo, el doble de la cultura vuelve siniestramente (ni el uno ni el otro, sino el impostor) para burlarse y minar, para perder el sentido del yo (self) magistral y su soberanía social. En este momento de "incertidumbre" intelectual y psíquica, la representación ya no puede garantizar la autoridad de la cultura, y la cultura ya no puede garantizar que sus sujetos "humanos" sean signos de humanidad. ${ }^{14}$

La favela crece y crece, y sus moradores por mucho que sean trasladados no dejan de encontrar nuevos locales para habitar hasta acabar invadiendo la ciudad, siendo imposibles de ocultar, esto es evidente en una ciudad como Río. Por eso la favela interroga solo con su presencia las contradicciones de un sistema que trata de autoafirmarse sin fisuras, evidencia sus fracasos.

Jacques realiza una crítica a la arquitectura actual por no respetar los movimientos de la ciudad. Un ejemplo límite podría ser los planes de urbanización de las favelas por considerar que, en verdad, los actuales conceptos de arquitectura no pueden sino petrificar o redirigir un flujo de energía, unas corrientes que ya están activas y activadas en sus calles. Su patrimonio, y no por casualidad también el de la obra de Oiticica, es el del movimiento, ser un espacio-movimiento del que los habitantes participan, tanto al construir sus casas como al inventarse cada uno de los espacios que usan, una característica imposible de museificar:

El habitante o el simple visitante, en el espacio-movimiento, se transforma siempre en actor, coautor o participante, así como los espectadores pasan a ser participantes y a veces incluso coautores de las obras de Oiticica, que en gran mayoría son verdaderos espacios-movimiento propuestos por el artista; en palabras de Oiticica: invitaciones para "vivencias". ${ }^{15}$ 
Digamos que Oiticica presenta la copia sucia del exotismo, del mundo capitalista, de las formas de habitar occidentales, de su urbanidad, de la postal turística. Además, presenta el resultado de una mimesis, que genera la hibridez, la de la favela. Si Bhabha está hablando de las relaciones colono/colonizado para explicar que de ellas resulta una cultura híbrida, desautorizada, Oiticica traslada esta misma operación a las relaciones del discurso hegemónico con la pobreza y la poscolonialidad tanto a nivel de representación como de urbanidad. La favela es este espacio casi igual pero no exactamente menos que uno y doble. Se construye desde un concepto de ciudad específica pero su espontaneidad le lleva a generar complejísimos espacios precarios, orgánicos, que nunca dejan de crecer. Es un híbrido, pero insisto, híbrido en Bhabha no significa solo mezcla, sino desautorización de los saberes hegemónicos, caricatura de un original que se verá desbordado ante la alteridad de su representación mimetizada, "que crea confusión entre opuestos" una y otra vez.
María Iñigo Clavo

¡Sucia Copia!
María Iñigo Clavo es investigadora, curadora y artista, con doctorado en Bellas Artes por la Universidad Complutense de Madrid, España, durante tres años sostuvo una residencia posdoctoral en la Universidade de São Paulo, Brasil.
Artigo recebido em 15 de maio de 2017 e aceito em 5 de julho de 2017. 